\title{
Single-Port Laparoscopic Total Extraperitoneal Inguinal Hernia Repair without Fixation of the Mesh
}

\author{
Sung Uk Bae, M.D. ${ }^{1}$, Byung Soh Min, M.D. ${ }^{2}$, Seung Hyuk Baik, M.D. ${ }^{2}$, Nam Kyu Kim, M.D. ${ }^{2}$, Hyuk Hur, M.D. ${ }^{2}$ \\ Division of Colorectal Surgery, Department of Surgery, 'School of Medicine, Keimyung University and Dongsan Medical Center, Daegu, ${ }^{2}$ Colorectal Cancer \\ Clinic, Severance Hospital, Yonsei University College of Medicine, Seoul, Korea
}

Purpose: Single-port laparoscopic surgery has been used increasingly in treatment of a variety of conditions, and one recently introduced technique is single-port laparoscopic total extraperitoneal (SPLTEP) hernia repair. The aim of this study was to evaluate the safety and technical feasibility of SPLTEP hernia repair without fixation of the mesh.

Methods: From June 2010 to October 2012, 112 consecutive patients underwent SPLTEP hernia repair. Results: We performed 129 inguinal hernia repairs in 112 patients, 17 were bilateral and 95 unilateral. There were 107 indirect hernias (83.0\%), 20 direct hernias, and 2 hernias had both of types. Successful SPLTEP hernia repair was performed in 110 patients, with two conversions to an open surgical approach. Median operative times for unilateral and bilateral hernias were 70 minutes (40 145 minutes) and 90 minutes (67 135 minutes), respectively, and the hospital stay was 1 day (1 5 days). Postoperative complications included 10 patients with wound seromas or hematomas, 2 with urinary retention, 4 with groin pain, and 1 with bleeding from the incision site. There were 3 early recurrences (2.4\%) during a median follow-up period of 42 months (range, 6 55).

Conclusion: SPLTEP inguinal hernia repair without fixation of the mesh is both safe and technically feasible, and provides acceptable operative outcomes. Conduct of a prospective randomized study with long-term follow-up is needed to confirm the benefit of this technique in patients with inguinal hernia.

Keywords: Hernia, Inguinal herniorrhaphy, Laparoscopy

This is an Open Access article distributed under the terms of the Creative Commons Attribution Non-Commercial License (http:// creativecommons.org/licenses/by-nc/4.0/) which permits unrestricted non-commercial use, distribution, and reproduction in any medium, provided the original work is properly cited.
Received October 2, 2015

Revised 1st November 25, 2015

2nd December 14, 2015

Accepted December 29, 2015

Corresponding author

Hyuk Hur

Division of Colorectal Surgery, Department of Surgery, Colorectal Cancer Clinic, Severance Hospital, Yonsei University College of Medicine, 50 Yonsei-ro, Seodaemun-gu, Seoul 41931, Korea Tel: +82-2-2228-2139 Fax: +82-2-313-8289 E-mail: hhyuk@yuhs.ac

\section{INTRODUCTION}

Laparoscopic inguinal hernia repair was first introduced in 1990, and the technique has since become increasingly popular worldwide. ${ }^{1}$ Compared with open repairs, laparoscopic inguinal hernia repair is associated with less postoperative pain, shorter recovery times, and improved cosmetic results. ${ }^{2}$ Although laparoscopic hernia repair was somewhat controversial due to its high recurrence rates and rare but dangerous complications, such as bowel or vascular injuries in early studies, recent studies have reported no difference in the recurrence rates of laparoscopic hernia repair and open tension-free repair for experienced surgeon. ${ }^{3,4}$ With minimally invasive surgery becoming increasingly common, questions of how to minimize surgical trauma and improve cosmetic outcomes have evolved as important topics of discussion. Each laparoscopic incision for trocar insertion presents risks for incisional hernias, bowel or vascular injuries, and unsatisfac- 
tory cosmetic outcomes. As a result, single-port laparoscopic surgery (SPLS) has been developed for treatment of a variety of conditions.

SPLS was first described in 1998 for laparoscopic appendectomy $^{5}$ and cholecystectomy, ${ }^{6}$ the SPLS technique has been incorporated into a variety of laparoscopic procedures. Since the first report of single-port laparoscopic total extraperitoneal (SPLTEP) hernia repair, there have been several studies in the published literature. ${ }^{8-11}$ The aim of this study was to evaluate the safety and technical feasibility of SPLTEP hernia repair without fixation of the mesh.

\section{MATERIALS AND METHODS}

Between June 2010 and October 2012, 112 consecutive patients underwent elective inguinal hernia repair by a single surgeon (H.H) at Severance Hospital, Yonsei University College of Medicine. All inguinal hernia repairs was planned to be performed by single-port laparoscopic approach. Prospectively collected data included age, gender, body mass index, hernia characteristics, gender, operative time, length of hospital stay, and perioperative complications. Postoperative wound pain was measured using the visual analog scale (VAS) pain score system with endpoints labeled as "no pain" (0-mm) and "worst possible pain" (100-mm) on postoperative day 1 . For the subgroup analysis, the patients were separated into 2 groups, unilateral and bilateral hernia repairs.

\section{Surgical technique of SPLTEP hernia repair without fixation}

All patients were given prophylactic antibiotics, and surgical procedures were carried out under general anesthesia with the patient in the supine position. A single $2.5-\mathrm{cm}$ vertical incision was made through the umbilical skin. The subcutaneous tissue was dissected to expose the linea alba. The linea alba was then incised vertically and the peritoneum was preserved.

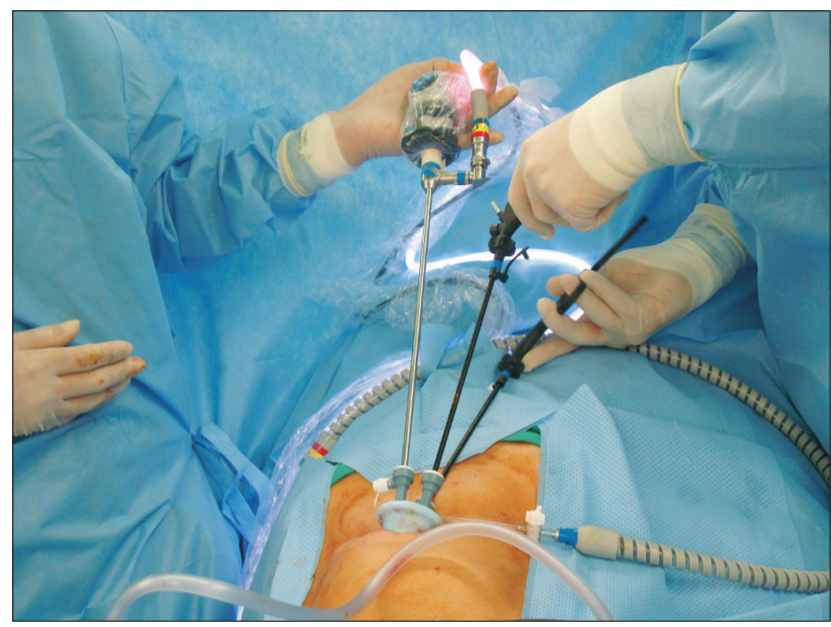

Fig. 1. Single-port with the camera inserted into the 5-mm port along with laparoscopic instruments.
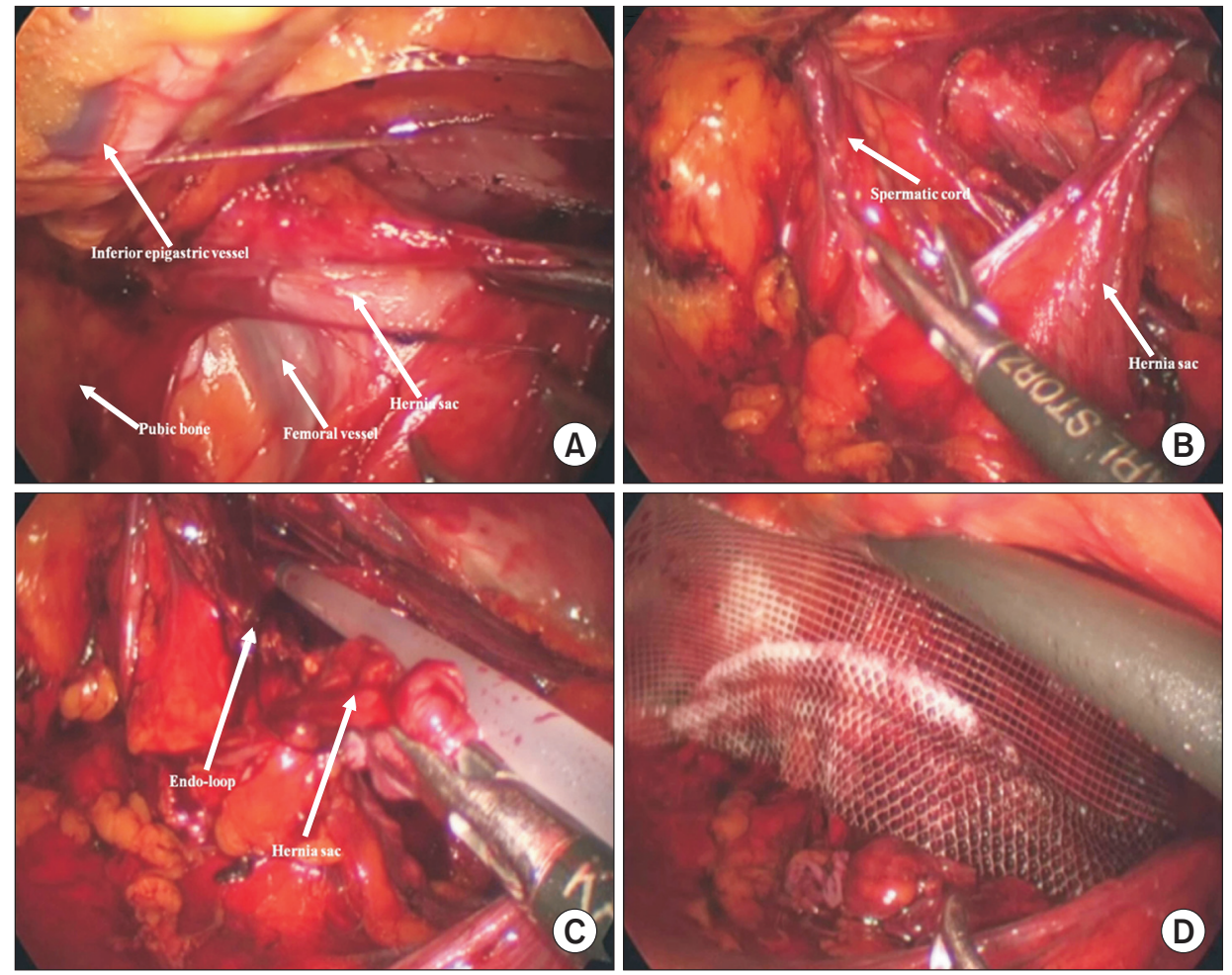

Fig. 2. Intraoperative views during a SPLTEP inguinal hernia repair. (A) Identification of the inferior epigastric vessels, symphysis pubis, femoral vessels, and hernia sac. (B) Isolation of the hernia sac from the spermatic cord. (C) Ligation of hernia sac just beyond the internal ring with a endoloop. (D) Placement of polyester mesh without fixation suturing. 
Extraperitoneal space was carefully generated by blunt finger dissection. A disposable SILS ${ }^{\mathrm{TM}}$ Multiple Instrument Access Port (Covidien, Mansfield, MA, USA) was placed in the preperitoneal space through the umbilical incision, and three 5-mm channels were inserted into each hole of the singleport. After $\mathrm{CO}_{2}$ insufflation via the insufflation channel of the single-port, an endoscopic camera 5-mm in diameter and 45$\mathrm{cm}$ in length along with graspers and monopolar diathermy were used to generate preperitoneal space (Fig. 1). Lateral space was dissected toward the anterior superior iliac spine and continued medially until the inferior epigastric vessels, symphysis pubis, and cord structures were identified (Fig. 2A). If a direct hernia was present, the hernia sac was reduced using gentle traction on the sac. In cases of indirect hernias, the hernia sac was carefully isolated, freed from the spermatic cord, and reduced from the internal ring by gentle traction and dissection (Fig. 2B). Indirect hernia sacs were routinely ligated just beyond the internal ring with a Vicryl Endoloop (Ethicon, Somerville, NJ, USA) and divided using endoshears (Fig. 2C). One 5-mm access channel was then removed and a $12-\mathrm{mm}$ access channel was subsequently inserted. Parietex Anatomical mesh (Covidien, Dublin, Ireland) $9-\mathrm{cm} \times 13-\mathrm{cm}$ in size was

Table 1. Patient and inguinal hernia characteristics

\begin{tabular}{|c|c|}
\hline Patient number & 112 \\
\hline \multicolumn{2}{|l|}{ Sex, n $1 \%)$} \\
\hline Male & $102(91)$ \\
\hline Female & $10(9)$ \\
\hline Age (yr), median (range) & $58(19 \sim 85)$ \\
\hline $\mathrm{BMI}\left(\mathrm{kg} / \mathrm{m}^{2}\right)$, median (range) & $22.7(16.8 \sim 36.7)$ \\
\hline \multicolumn{2}{|l|}{ ASA classification, n (\%) } \\
\hline 1 & $85(76)$ \\
\hline 2 & $23(21)$ \\
\hline 3 & $4(4)$ \\
\hline 4 & 0 \\
\hline \multicolumn{2}{|l|}{ Site of hernia, n (\%) } \\
\hline Right inguinal & $59(53)$ \\
\hline Left inguinal & $36(32)$ \\
\hline Bilateral & $17(15)$ \\
\hline \multicolumn{2}{|l|}{ Type of hernia, n (\%) } \\
\hline Indirect hernia & 107 (83.0) \\
\hline Direct hernia & $20(15.5)$ \\
\hline Indirect and direct hernia & $2(1.5)$ \\
\hline
\end{tabular}

ASA = American Society of Anesthesiologists; $\mathrm{BMI}=$ body mass index. introduced through the 12- $\mathrm{mm}$ access channel, unfolded, and placed around the spermatic cord from the pubic symphysis to the anterior iliac spine laterally without fixation suturing or fibrin sealant (Fig. 2D). After the operation was completed, the space was deflated under direct visualization without drainage.

\section{Statistical analyses}

The statistical analyses were performed with IBM SPSS 20.0 software (SPSS Inc., Chicago, IL, USA). Clinical characteristics and variables were compared between bilateral and unilateral groups by an independent-sample t-test for continuous data or a chi-square test for categorical data. A $p$ value $<0.05$ was considered to be statistically significant.

\section{RESULTS}

\section{Patient characteristics}

We performed 129 inguinal hernia repairs in 112 patients, 17 of which were bilateral and 95 unilateral. There were no femoral or scrotal hernias. Patient demographics and hernia characteristics are summarized in Table 1. The median age of the patients was 58 years (range, 19 85 years), with 10 women and 102 men and the body mass index was $22.7 \mathrm{~kg} / \mathrm{m}^{2}$ $\left(16.8 \sim 36.7 \mathrm{~kg} / \mathrm{m}^{2}\right)$. There were 107 indirect henias (83.0\%), 20

Table 2. Operative outcomes and morbidity after SPLTEP inguinal hernia repair

\begin{tabular}{ll}
\hline Operation time (min), median (range) & \\
Unilateral & $70(40 \sim 145)$ \\
Bilateral & $90(67 \sim 135)$ \\
\hline VAS score on POD 1, median (range) & $2(1 \sim 7)$ \\
\hline Hospital days (days), median (range) & $1(1 \sim 5)$ \\
\hline Conversion, n (\%) & $2(1.8)$ \\
\hline Postoperative complications, n (\%) & $17(15.3)$ \\
Wound hematoma & $4(3.6)$ \\
Wound seroma & $6(5.4)$ \\
Urinary retention & $2(1.8)$ \\
Groin pain & $4(3.6)$ \\
Wound bleeding & $1(0.9)$ \\
\hline Mortality, n (\%) & $0(0)$ \\
Recurrence, n (\%) & $3(2.4 \%)$ \\
\hline
\end{tabular}

SPLTEP = single-port laparoscopic totally extraperitoneal; VAS = visual analog scale;, POD = postoperative day. 
direct hernias, and 2 hernias had both of types.

\section{Perioperative clinical outcomes}

Successful SPLTEP hernia repair was performed in 110 patients, with 2 conversions to an open surgical approach (Table 2). Both conversions were due to peritoneal tears that occurred as the surgeon created preperitoneal space. Median operative times for unilateral and bilateral hernias were 70 minutes (40 145 minutes) and 90 minutes (67 135 minutes),

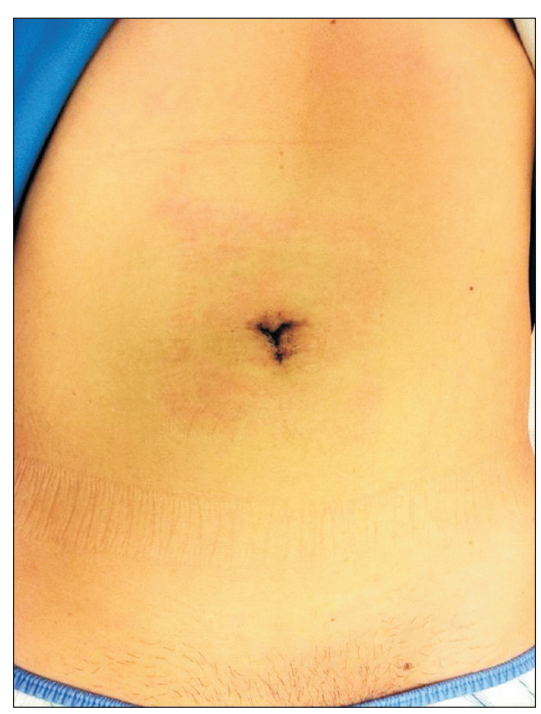

Fig. 3. Operativescar on postoperative day 7 after SPLTEP inguinal hernia repair. respectively. The median postoperative hospital stay was 1 day ( $1 \sim 5$ days) and the median pain score (VAS score) on postoperative day 1 was $2(1 \sim 7)$. Cosmetic results were excellent, with the $2.5-\mathrm{cm}$ single-incision being inside the natural fold of the umbilicus (Fig. 3). All procedures were completed without major intraoperative complications, though postoperative complications included 10 patients with wound seromas or hematomas, 2 with urinary retention, 4 with groin pain, and 1 with bleeding from the incision site. All complications were managed with conservative treatment. There were 3 early recurrences $(2.4 \%)$ during a median follow-up period of 42 months (range, 6 55).

\section{Subgroup analysis of unilateral versus bilateral inguinal hernia repair}

Demographic characteristics were similar between the two groups for age, gender, and ASA classification (Table 3). The mean operative time was significantly longer in the bilateral group (95.5 \pm 21.6 minutes) than in the unilateral group (73.5 \pm 18.4 minutes; $p<0.001$ ). Pain scores for the incision site on postoperative day 1 showed no significant differences between the two groups (bilateral: 2.2 \pm 1.2 ; unilateral: $2.2 \pm$ $0.9 ; p=0.894)$. Morbidity within 30 days after surgery and the length of hospital stay were comparable between the two groups.

\section{DISCUSSION}

The present study demonstrates that, SPLTEP repairs can be

Table 3. Subgroup analysis of unilateral versus bilateral inguinal hernia repair

\begin{tabular}{|c|c|c|c|}
\hline Variables & Unilateral group $(n=17)$ & Bilateral group $(n=95)$ & $p$ value \\
\hline Age (yr), mean $\pm S D$ & $54.0 \pm 18.43$ & $59.4 \pm 16.6$ & 0.258 \\
\hline $\operatorname{Sex}|\%|$ & & & 0.656 \\
\hline Male & $87(91.6)$ & $15(88.2)$ & \\
\hline Female & $8(8.4)$ & 2 (11.8) & \\
\hline ASA classification, $(\%)$ & & & 0.393 \\
\hline 1 & $70(73.7)$ & $15(88.2)$ & \\
\hline 2 & $21(22.1)$ & $2(11.8)$ & \\
\hline 3 & $4(4.2)$ & $0(0)$ & \\
\hline Operation time (min), median (range) & $73.5 \pm 18.4$ & $95.5 \pm 21.6$ & $<0.001$ \\
\hline VAS score on POD 1, mean \pm SD & $2.2 \pm 0.9$ & $2.2 \pm 1.2$ & 0.894 \\
\hline Postoperative complications, n (\%) & $13(13.7)$ & $3(17.6)$ & 0.667 \\
\hline Hospital days (days), median (range) & $1.2 \pm 0.7$ & $1.1 \pm 0.3$ & 0.537 \\
\hline
\end{tabular}

$S D=$ standard deviation; $A S A=$ American Society of Anesthesiologists; $V A S=$ visual analog scale; $P O D=$ postoperative day. 
performed with safety, feasibility, and good technical efficiency for patients with inguinal hernia. The surgical outcomes of the present case series are consistent with our hypothesis that by using the single-port for TEP repair without fixation of the mesh, high postoperative pain can be prevented for inguinal hernia repair surgery, especially for patients with both inguinal hernias. In a literature review of the most recent data from SPLTEP inguinal hernia repairs, Minh Do et al. ${ }^{12}$ reported operative times ranging from 30 to 100 minutes for unilateral hernia repair and 26 to 120 minutes for bilateral hernia repair. Our results showed comparable median operating times of 70 minutes for unilateral and 90 minutes for bilateral hernia repair, with only two conversions to tension-free Lichtenstein repair.

Laparoscopic inguinal hernia repair was first used in the 1990s by Ger et al. ${ }^{1}$ At that time, this technique was controversial due to its relative technical difficultly compared with open hernia repair. Additionally, some studies reported postsurgical recurrence rates that were as high as $25 \%{ }^{13,14}$ However, after more than a decade of experience with laparoscopic hernia repair, recurrence rates have decreased and this technique has become the treatment of choice at many institutions. The main advantages of laparoscopic inguinal hernia repair are less postoperative pain, shorter recovery times, and improved cosmetic results. This method has been well established because of its safety, efficacy, and patient satisfaction. There are two well-described approaches to laparoscopic inguinal hernia repair including the total extraperitoneal (TEP) and the transabdominal preperitoneal (TAPP) methods. TEP repair is preferred to TAPP repair by some surgeons because it does not require entry into the peritoneal cavity, and thus avoids the risk of mesh coming into contact with intra-abdominal structures. ${ }^{15}$

As minimally invasive surgery becomes more common, questions of how to minimize surgical trauma and improve cosmetic outcomes have evolved as important topics of discussion. For this reason, SPLS is being increasingly used to treat a variety of conditions. Since SPLS was first described in 1998 for laparoscopic appendectomy ${ }^{5}$ and cholecystectomy, ${ }^{6}$ the feasibility of SPLS has been reported for a number of surgical procedures including, ${ }^{12}$ colectomy, ${ }^{16}$ adrenalectomy,${ }^{17}$ nephrectomy, ${ }^{18}$ and splenectomy. ${ }^{19}$ The potential advantages of this approach are improved cosmetic outcomes, less postoperative incisional pain, fewer wound complications, and reduction of the number of trocars used. Since the first (SPLTEP) hernia repair was reported by Filipovic-Cugura et al., ${ }^{7}$ and there have been few related reports in the literature. ${ }^{8-10}$

In this study, median VAS score on postoperative day 1 was 2 this result was comparable to previous studies of SPLTEP with tack placement or suturing ${ }^{20-22}$ Although we did not com- pare the pain score of SPLTEP without fixation with that of SPLTEP with tack placement, the present study demonstrated that SPLTEP repair without fixation suturing of the mesh did not seem to be related to high levels of postoperative pain. We unfolded the mesh without fixation suturing before the preperitoneal space was deflated under direct visualization. In the laparoscopic approach, various techniques of mesh attachment have been described as being in favor of fixation of the mesh used in SPLTEP hernia repairs. ${ }^{9,23}$ However, Belyansky et al. ${ }^{4}$ demonstrated that bilateral inguinal hernia repairs were associated with higher pain levels during the early postoperative time period when compared to unilateral repairs and that the use of more than 10 tacks on each side in bilateral repairs was associated with a higher frequency of symptomatic patients. In our study, to investigate whether bilateral SPLTEP repair results in higher pain levels, we compared the outcomes of the bilateral and unilateral inguinal hernia groups. Pain scores for incision site on postoperative day 1 showed no significant differences between two groups. These results suggest that SPLTEP repair without fixation suturing of the mesh is not be related to high levels of postoperative pain. Messaris et al. ${ }^{24}$ have also suggested that mesh in the preperitoneal space does not need to be anchored and is instead held in place by the intra-abdominal pressure generated by the abdominal cavity viscera. There may be benefits of non-fixation with respect to shorter operative times, cost reduction, less postoperative pain, and fewer complications related to the introduction of foreign material. Due to concerns for nerve entrapment or potential tissue injury by tacks or suturing, we suggest an alternative means of securing the mesh: non-fixation of the mesh.

In the present study, there were 3 recurrences (2.4\%) during a median follow-up period of 42 months (range, 6 55). These recurrences occurred within first 10 cases during the learning curve period and were associated with inappropriate placement of mesh. Schwab et al. ${ }^{25}$ reported that there were 2 recurrences (2.3\%) in the fibrin sealant group and 5 recurrences (5.7\%) in the stapled group in patients who underwent laparoscopic TEP repair for inguinal hernia. Topart et al. ${ }^{26}$ demonstrated that 3 recurrences $(2.9 \%)$ in tack staples group and 1 recurrence $(1.5 \%)$ in fibrin glue group occurred in 66 patients. These results suggest that SPLTEP repair without fixation suturing of the mesh in our study is not related to high recurrence rate.

SPLS has its own unique challenges including the relative loss of triangulation, inline vision, clashing of instruments, and a steep learning curve for mastering the procedure. To overcome these technical challenges, OnderSurgit ${ }^{23}$ recom- $^{-}$ mended the "crossed instrument technique," and Argawalet al. used articulated laparoscopic instruments. In addition, Hanh $\operatorname{Tran}^{27}$ suggested two maneuvers including "inline" dissection in which one dissecting instrument is pushing away while the 
other is pulling outward, and "vertical" dissection in which the instruments move vertically in opposite directions on either side of the laparoscope. Also, significant coordination between the camera assistant and the operator surgeon is important to avoid clashing of the instruments and the laparoscope during the operation. In this study, we used an $30^{\circ}$ endoscopic camera $5-\mathrm{mm}$ in diameter and $45-\mathrm{cm}$ in length to minimize the collision between camera and instruments.

In recent years, the focus in hernia repair has begun to shift from minimizing the recurrence rate to quality of life, including postoperative pain and cosmetic results, which are two goals of minimally invasive surgery. However, the cosmetic result after hernia repair is generally ignored by many surgeons. The cosmetic result of the single incision after SPLTEP repair hidden in the normal groove of the umbilicus seems to be excellent. However, a prospective study including an assessment of cosmetic appearance and body image using a questionnaire is needed in the future.

Our study has several limitations, including its retrospective nature and its lack of both a control group and registration of a scar assessment questionnaire. From the limited information from a single center, we can suggest the following advantages of single incision laparoscopic total extraperitoneal inguinal hernia repair without fixation of the mesh:

1. Allows safe and feasible repair of inguinal hernia

2. Presents excellent cosmetic outcomes with nearly scarless incision

3. Results in less postoperative pain than the mesh anchoring technique

In conclusion, SPLTEP inguinal hernia repair without fixation of the mesh is both safe and technically feasible, and provides acceptable operative outcomes. A prospective randomized study with long-term follow-up is needed to confirm the benefit of this technique in patients with inguinal hernia.

\section{REFERENCES}

1) Ger R, Monroe K, Duvivier R, Mishrick A. Management of indirect inguinal hernias by laparoscopic closure of the neck of the sac. Am J Surg 1990;159:370-373.

2) Memon MA, Cooper NJ, Memon B, Memon MI, Abrams KR. Meta-analysis of randomized clinical trials comparing open and laparoscopic inguinal hernia repair. Br J Surg 2003;90:1479-1492.

3) Langeveld HR, van't Riet M, Weidema WF, et al. Total extraperitoneal inguinal hernia repair compared with Lichtenstein (the LEVEL-Trial): a randomized controlled trial. Ann Surg 2010;251: 819-824.

4) Belyansky I, Tsirline VB, Klima DA, Walters AL, Lincourt AE, Heniford TB. Prospective, comparative study of postoperative quality of life in TEP, TAPP, and modified Lichtenstein repairs.
Ann Surg 2011;254:709-714; discussion 714-705.

5) Esposito C. One-trocar appendectomy in pediatric surgery. Surg Endosc 1998;12:177-178.

6) Piskun G, Rajpal S. Transumbilical laparoscopic cholecystectomy utilizes no incisions outside the umbilicus. J Laparoendosc Adv Surg Tech A 1999;9:361-364.

7) Filipovic-Cugura J, Kirac I, Kulis T, Jankovic J, Bekavac-Beslin M. Single-incision laparoscopic surgery (SILS) for totally extraperitoneal (TEP) inguinal hernia repair: first case. Surg Endosc 2009;23:920-921.

8) Agrawal S, Shaw A, Soon Y. Single-port laparoscopic totally extraperitoneal inguinal hernia repair with the TriPort system: initial experience. Surg Endosc 2010;24:952-956.

9) Chung SD, Huang CY, Wang SM, et al. Laparoendoscopic singlesite totally extraperitoneal adult inguinal hernia repair: initial 100 patients. Surg Endosc 2011;25:3579-3583.

10) Kim JH, Park SM, Kim JJ, Lee YS. Initial experience of single port laparoscopic totally extraperitoneal hernia repair: nearlyscarless inguinal hernia repair. J Korean Surg Soc 2011;81:339343.

11) Tsai YC, Ho CH, Tai HC, Chung SD, Chueh SC. Laparoendoscopic single-site versus conventional laparoscopic total extraperitoneal hernia repair: a prospective randomized clinical trial. Surg Endosc 2013;27:4684-4692.

12) Do M, Liatsikos E, Beatty J, et al. Laparoendoscopic single-site extraperitoneal inguinal hernia repair: initial experience in $10 \mathrm{pa}^{-}$ tients. J Endourol 2011;25:963-968.

13) Fitzgibbons RJ Jr, Camps J, Cornet DA, et al. Laparoscopic inguinal herniorrhaphy. Results of a multicenter trial. Ann Surg 1995;221:3-13.

14) Vogt DM, Curet MJ, Pitcher DE, Martin DT, Zucker KA. Preliminary results of a prospective randomized trial of laparoscopic onlay versus conventional inguinal herniorrhaphy. Am J Surg 1995;169:84-89; discussion 89-90.

15) Wake BL, McCormack K, Fraser C, Vale L, Perez J, Grant AM. Transabdominal pre-peritoneal (TAPP) vs totally extraperitoneal (TEP) laparoscopic techniques for inguinal hernia repair. Cochrane Database Syst Rev 2005:CD004703.

16) Ramos-Valadez DI, Patel CB, Ragupathi M, Bartley Pickron $T$, Haas EM. Single-incision laparoscopic right hemicolectomy: safety and feasibility in a series of consecutive cases. Surg Endosc 2010;24:2613-2616.

17) Chung SD, Huang CY, Wang SM, Tai HC, Tsai YC, Chueh SC. Laparoendoscopic single-site (LESS) retroperitoneal adrenalectomy using a homemade single-access platform and standard laparoscopic instruments. Surg Endosc 2011;25:1251-1256.

18) Raman JD, Bagrodia A, Cadeddu JA. Single-incision, umbilical laparoscopic versus conventional laparoscopic nephrectomy: a comparison of perioperative outcomes and short-term measures of convalescence. Eur Urol 2009;55:1198-1204. 
19) Targarona EM, Pallares JL, Balague C, et al. Single incision approach for splenic diseases: a preliminary report on a series of 8 cases. Surg Endosc 2010;24:2236-2240.

20) Kim JH, An CH, Lee YS, Kim HY, Lee JI. Single incision laparoscopic totally extraperitoneal hernioplasty (SIL-TEP): experience of 512 procedures. Hernia 2015;19:417-422.

21) Tran H, Turingan I, Tran K, Zajkowska M, Lam V, Hawthorne W. Potential benefits of single-port compared to multiport laparoscopic inguinal herniorraphy: a prospective randomized controlled study. Hernia 2014;18:731-744.

22) Kim JH, Lee YS, Kim JJ, Park SM. Single port laparoscopic totally extraperitoneal hernioplasty: a comparative study of short-term outcome with conventional laparoscopic totally extraperitoneal hernioplasty. World J Surg 2013;37:746-751.

23) Surgit O. Single-incision Laparoscopic surgery for total extra- peritoneal repair of inguinal hernias in 23 patients. Surg Laparosc Endosc Percutan Tech 2010;20:114-118.

24) Messaris E, Nicastri G, Dudrick SJ. Total extraperitoneal laparoscopic inguinal hernia repair without mesh fixation: prospective study with 1-year follow-up results. Arch Surg 2010;145:334-338.

25) Schwab R, Willms A, Kroger A, Becker HP. Less chronic pain following mesh fixation using a fibrin sealant in TEP inguinal hernia repair. Hernia 2006;10:272-277.

26) Topart P, Vandenbroucke F, Lozac'h P. Tisseel versus tack staples as mesh fixation in totally extraperitoneal laparoscopic repair of groin hernias: a retrospective analysis. Surg Endosc 2005;19:724727.

27) Tran H. Safety and efficacy of single incision laparoscopic surgery for total extraperitoneal inguinal hernia repair. JSLS 2011;15:4752. 